\title{
Article \\ Equivalent Properties of Two Kinds of Hardy-Type Integral Inequalities
}

\author{
Michael Th. Rassias ${ }^{1,2,3, *}$, Bicheng Yang ${ }^{4}$ and Andrei Raigorodskii ${ }^{2,5,6,7}$ \\ 1 Institute of Mathematics, University of Zurich, $\mathrm{CH}-8057$ Zurich, Switzerland \\ 2 Moscow Institute of Physics and Technology, Institutskiy per, d. 9, 141700 Dolgoprudny, Russia; \\ raigorodsky@yandex-team.ru \\ 3 Program in Interdisciplinary Studies, Institute for Advanced Study, 1 Einstein Drive, Princeton, NJ 08540, \\ USA \\ 4 Department of Mathematics, Guangdong University of Education, Guangzhou 510303, China; \\ bcyang@gdei.edu.cn \\ 5 Faculty of Bioengineering and Bioinformatics, Moscow State University, 119991 Moscow, Russia \\ 6 Institute of Mathematics and Computer Science, Buryat State University, 670000 Ulan-Ude, Russia \\ 7 Caucasus Mathematical Center, Adyghe State University, 385012 Maykop, Russia \\ * Correspondence: michail.rassias@math.uzh.ch
}

check for

updates

Citation: Rassias, M.T.; Yang, B.;

Raigorodskii, A. Equivalent

Properties of Two Kinds of

Hardy-Type Integral Inequalities.

Symmetry 2021, 13, 1006. https://

doi.org/10.3390/sym13061006

Academic Editor: Ioan Rașa

Received: 13 May 2021

Accepted: 1 June 2021

Published: 4 June 2021

Publisher's Note: MDPI stays neutral with regard to jurisdictional claims in published maps and institutional affiliations.

Copyright: (C) 2021 by the authors. Licensee MDPI, Basel, Switzerland. This article is an open access article distributed under the terms and conditions of the Creative Commons Attribution (CC BY) license (https:/ / creativecommons.org/licenses/by/ $4.0 /)$.

\begin{abstract}
In this paper, using weight functions as well as employing various techniques from real analysis, we establish a few equivalent conditions of two kinds of Hardy-type integral inequalities with nonhomogeneous kernel. To prove our results, we also deduce a few equivalent conditions of two kinds of Hardy-type integral inequalities with a homogeneous kernel in the form of applications. We additionally consider operator expressions. Analytic inequalities of this nature and especially the techniques involved have far reaching applications in various areas in which symmetry plays a prominent role, including aspects of physics and engineering.
\end{abstract}

Keywords: Hardy-type integral inequality; weight function; equivalent form; operator; norm

MSC: 26D15; 47A05

\section{Introduction}

In 1925, by introducing one pair of conjugate exponents $(p, q)$, Hardy [1] established a well-known extension of Hilbert's integral inequality as follows.

If $p>1, \frac{1}{p}+\frac{1}{q}=1, f(x), g(y) \geq 0$,

$$
0<\int_{0}^{\infty} f^{p}(x) d x<\infty \text { and } 0<\int_{0}^{\infty} g^{q}(y) d y<\infty,
$$

then:

$$
\int_{0}^{\infty} \int_{0}^{\infty} \frac{f(x) g(y)}{x+y} d x d y<\frac{\pi}{\sin (\pi / p)}\left(\int_{0}^{\infty} f^{p}(x) d x\right)^{\frac{1}{p}}\left(\int_{0}^{\infty} g^{q}(y) d y\right)^{\frac{1}{q}},
$$

where the constant factor $\frac{\pi}{\sin (\pi / p)}$ is the best possible.

Inequalities (1) as well as Hilbert's integral inequality (for $p=q=2$ in (1), cf. [2]) are important in analysis and its applications (cf. [3,4]).

Almost ten years later, in 1934, Hardy et al. proved an extension of (1) with the general homogeneous kernel of degree -1 as $k_{1}(x, y)$ (cf. [3], Theorem 319). The following Hilbert-type integral inequality with the general nonhomogeneous kernel was established.

If $h(u)>0, \phi(\sigma)=\int_{0}^{\infty} h(u) u^{\sigma-1} d u \in \mathbf{R}_{+}$, then: 


$$
\begin{aligned}
& \int_{0}^{\infty} \int_{0}^{\infty} h(x y) f(x) g(y) d x d y \\
< & \phi\left(\frac{1}{p}\right)\left(\int_{0}^{\infty} x^{p-2} f^{p}(x) d x\right)^{\frac{1}{p}}\left(\int_{0}^{\infty} g^{q}(y) d y\right)^{\frac{1}{q}},
\end{aligned}
$$

where the constant factor $\phi\left(\frac{1}{p}\right)$ is the best possible (cf. [3], Theorem 350).

In 1998, by introducing an independent parameter $\lambda>0$, Yang proved an extension of Hilbert's integral inequality with the kernel $\frac{1}{(x+y)^{\lambda}}$ (cf. [5,6]). In 2004, by introducing another pair of conjugate exponents $(r, s)$, Yang [7] was able to estabish an extension of (1) with the kernel $\frac{1}{x^{\lambda}+y^{\lambda}}(\lambda>0)$. In the paper [8], a further extension of (1) was proved along with the result of the paper [5] with the kernel $\frac{1}{(x+y)^{\lambda}}$. Several papers (cf. [9-14]) provided some extensions of (1) with parameters. In 2009, Yang presented the following extension of (1) (cf. $[15,16])$.

If $\lambda_{1}+\lambda_{2}=\lambda \in \mathbf{R}=(-\infty, \infty), k_{\lambda}(x, y)$ is a non-negative homogeneous function of degree $-\lambda$, satisfying:

$$
k_{\lambda}(u x, u y)=u^{-\lambda} k_{\lambda}(x, y)(u, x, y>0),
$$

and:

$$
k\left(\lambda_{1}\right)=\int_{0}^{\infty} k_{\lambda}(u, 1) u^{\lambda_{1}-1} d u \in \mathbf{R}_{+}=(0, \infty),
$$

then we have:

$$
\begin{aligned}
& \int_{0}^{\infty} \int_{0}^{\infty} k_{\lambda}(x, y) f(x) g(y) d x d y \\
< & k\left(\lambda_{1}\right)\left(\int_{0}^{\infty} x^{p\left(1-\lambda_{1}\right)-1} f^{p}(x) d x\right)^{\frac{1}{p}}\left(\int_{0}^{\infty} y^{q\left(1-\lambda_{2}\right)-1} g^{q}(y) d y\right)^{\frac{1}{q}},
\end{aligned}
$$

where the constant factor $k\left(\lambda_{1}\right)$ is the best possible.

For $\lambda=1, k_{\lambda}(x, y)=\frac{1}{x+y}, \lambda_{1}=\frac{1}{q}, \lambda_{2}=\frac{1}{p}$, (3) reduces to (1). The following extension of (2) was proven:

$$
\begin{aligned}
& \int_{0}^{\infty} \int_{0}^{\infty} h(x y) f(x) g(y) d x d y \\
< & \phi(\sigma)\left(\int_{0}^{\infty} x^{p(1-\sigma)-1} f^{p}(x) d x\right)^{\frac{1}{p}}\left(\int_{0}^{\infty} y^{q(1-\sigma)-1} g^{q}(y) d y\right)^{\frac{1}{q}},
\end{aligned}
$$

where the constant factor $\phi(\sigma)$ is the best possible (cf. [17]).

For $\sigma=\frac{1}{p}$, (4) reduces to (2).

Some equivalent inequalities of (3) and (4) are considered in [16]. In 2013, Yang [17] also studied the equivalency between (3) and (4) by adding a condition. In 2017, Hong [18] proved an equivalent condition between (3) and a few parameters. Some similar results were obtained in [19-28].

Remark 1 (cf. [17]). If $h(x y)=0$, for $x y>1$, then:

$$
\phi(\sigma)=\int_{0}^{1} h(u) u^{\sigma-1} d u=\phi_{1}(\sigma) \in \mathbf{R}_{+},
$$

and (4) reduces to the following Hardy-type integral inequality with nonhomogeneous kernel: 


$$
\begin{aligned}
& \int_{0}^{\infty} g(y)\left(\int_{0}^{\frac{1}{y}} h(x y) f(x) d x\right) d y \\
< & \phi_{1}(\sigma)\left(\int_{0}^{\infty} x^{p(1-\sigma)-1} f^{p}(x) d x\right)^{\frac{1}{p}}\left(\int_{0}^{\infty} y^{q(1-\sigma)-1} g^{q}(y) d y\right)^{\frac{1}{q}},
\end{aligned}
$$

where the constant factor $\phi_{1}(\sigma)$ is the best possible.

If $h(x y)=0$, for $x y<1$, then:

$$
\phi(\sigma)=\int_{1}^{\infty} h(u) u^{\sigma-1} d u=\phi_{2}(\sigma) \in \mathbf{R}_{+},
$$

and (4) reduces to the following kind of Hardy-type integral inequality with nonhomogeneous kernel:

$$
\begin{aligned}
& \int_{0}^{\infty} g(y)\left(\int_{\frac{1}{y}}^{\infty} h(x y) f(x) d x\right) d y \\
< & \phi_{2}(\sigma)\left(\int_{0}^{\infty} x^{p(1-\sigma)-1} f^{p}(x) d x\right)^{\frac{1}{p}}\left(\int_{0}^{\infty} y^{q(1-\sigma)-1} g^{q}(y) d y\right)^{\frac{1}{q}},
\end{aligned}
$$

where the constant factor $\phi_{2}(\sigma)$ is the best possible.

In this paper, using weight functions as well as employing various techniques from real analysis, we establish a few equivalent conditions of two kinds of Hardy-type integral inequalities with the nonhomogeneous kernel:

$$
\frac{|\ln x y|^{\beta}}{(x y)^{\lambda}+1}(\beta>-1, \lambda>0) .
$$

To prove our results, we also deduce a few equivalent conditions of two kinds of Hardy-type integral inequalities with a homogeneous kernel in the form of applications. We additionally consider operator expressions. Analytic inequalities of this nature and especially the techniques involved have far reaching applications in various areas in which symmetry plays a prominent role, including aspects of physics and engineering.

\section{Two Lemmas}

For $\beta>-1, \lambda>0$, we set

$$
h(u):=\frac{|\ln u|^{\beta}}{u^{\lambda}+1}(u>0) .
$$

For $\sigma>0$, by the Lebesgue term-by-term integration theorem, we derive that:

$$
\begin{aligned}
k_{1}(\sigma) & :=\int_{0}^{1} h(u) u^{\sigma-1} d u=\int_{0}^{1} \frac{(-\ln u)^{\beta}}{u^{\lambda}+1} u^{\sigma-1} d u \\
& =\int_{0}^{1}(-\ln u)^{\beta} \sum_{k=0}^{\infty}(-1)^{k} u^{k \lambda+\sigma-1} d u \\
& =\int_{0}^{1}(-\ln u)^{\beta} \sum_{i=0}^{\infty}\left(u^{2 i \lambda}-u^{(2 i+1) \lambda}\right) u^{\sigma-1} d u \\
& =\sum_{i=0}^{\infty} \int_{0}^{1}(-\ln u)^{\beta}\left(u^{2 i \lambda}-u^{(2 i+1) \lambda}\right) u^{\sigma-1} d u \\
& =\sum_{k=0}^{\infty}(-1)^{k} \int_{0}^{1}(-\ln u)^{\beta} u^{k \lambda+\sigma-1} d u .
\end{aligned}
$$


Setting $v=(k \lambda+\sigma)(-\ln u)$ in the above integral, we obtain:

$$
\begin{aligned}
k_{1}(\sigma) & =\sum_{k=0}^{\infty} \frac{(-1)^{k}}{(k \lambda+\sigma)^{\beta+1}} \int_{0}^{\infty} v^{\beta} e^{-v} d v \\
& =\frac{\Gamma(\beta+1)}{\lambda^{\beta+1}} \xi\left(\beta+1, \frac{\sigma}{\lambda}\right) \in \mathbf{R}_{+},
\end{aligned}
$$

where:

$$
\Gamma(\eta):=\int_{0}^{\infty} v^{\eta-1} e^{-v} d v(\eta>0)
$$

stands for the gamma function and:

$$
\xi(s, a):=\sum_{k=0}^{\infty} \frac{(-1)^{k}}{(k+a)^{s}}(\operatorname{Re}(s), a>0),
$$

which is a function very well known for its applications in analytic number theory.

For $0<\sigma<\lambda, \mu=\lambda-\sigma>0$, setting $v=\frac{1}{u}$, by (7), we obtain that:

$$
\begin{aligned}
k_{2}(\sigma): & =\int_{1}^{\infty} h(u) u^{\sigma-1} d u \\
& =\int_{1}^{\infty} \frac{(\ln u)^{\beta}}{u^{\lambda}+1} u^{\sigma-1} d u=\int_{0}^{1} \frac{(-\ln v)^{\beta}}{v^{\lambda}+1} v^{\mu-1} d v \\
& =\frac{\Gamma(\beta+1)}{\lambda^{\beta+1}} \xi\left(\beta+1, \frac{\mu}{\lambda}\right)=k_{1}(\mu) \in \mathbf{R}_{+} .
\end{aligned}
$$

In the sequel, we assume that $p>1, \frac{1}{p}+\frac{1}{q}=1, \sigma_{1}, \mu_{1} \in \mathbf{R}$.

Lemma 1. If $\beta>-1, \sigma, \lambda>0$, there exists a constant $M_{1}$, such that for any non-negative measurable functions $f(x)$ and $g(y)$ in $(0, \infty)$, the following inequality:

$$
\begin{aligned}
& \int_{0}^{\infty} g(y)\left[\int_{0}^{\frac{1}{y}} \frac{|\ln x y|^{\beta}}{(x y)^{\lambda}+1} f(x) d x\right] d y \\
\leq & M_{1}\left[\int_{0}^{\infty} x^{p(1-\sigma)-1} f^{p}(x) d x\right]^{\frac{1}{p}}\left[\int_{0}^{\infty} y^{q\left(1-\sigma_{1}\right)-1} g^{q}(y) d y\right]^{\frac{1}{q}}
\end{aligned}
$$

holds true. Then, we have $\sigma_{1}=\sigma$, and $M_{1} \geq k_{1}(\sigma)$.

Proof. If $\sigma_{1}>\sigma$, then for $n \geq \frac{1}{\sigma_{1}-\sigma}(n \in \mathbf{N})$, we set the following two functions:

$$
f_{n}(x):=\left\{\begin{array}{c}
x^{\sigma+\frac{1}{p n}-1}, 0<x \leq 1 \\
0, x>1
\end{array}, g_{n}(y):=\left\{\begin{array}{c}
0,0<y<1 \\
y^{\sigma_{1}-\frac{1}{q^{n}}-1}, y \geq 1
\end{array}\right.\right.
$$

and deduce that:

$$
J_{1}:=\left[\int_{0}^{\infty} x^{p(1-\sigma)-1} f_{n}^{p}(x) d x\right]^{\frac{1}{p}}\left[\int_{0}^{\infty} y^{q\left(1-\sigma_{1}\right)-1} g_{n}^{q}(y) d y\right]^{\frac{1}{q}}=n .
$$

Setting $u=x y$, we obtain: 


$$
\begin{aligned}
I_{1} & :=\int_{0}^{\infty} g_{n}(y)\left(\int_{0}^{\frac{1}{y}} \frac{|\ln x y|^{\beta}}{(x y)^{\lambda}+1} f_{n}(x) d x\right) d y \\
& =\int_{1}^{\infty}\left(\int_{0}^{\frac{1}{y}} \frac{(-\ln x y)^{\beta}}{(x y)^{\lambda}+1} x^{\sigma+\frac{1}{p n}-1} d x\right) y^{\sigma_{1}-\frac{1}{q^{n}}-1} d y \\
& =\int_{1}^{\infty} y^{\left(\sigma_{1}-\sigma\right)-\frac{1}{n}-1} d y \int_{0}^{1} \frac{(-\ln u)^{\beta}}{u^{\lambda}+1} u^{\sigma+\frac{1}{p n}-1} d u
\end{aligned}
$$

and then by (9), we have:

$$
\begin{aligned}
& \int_{1}^{\infty} y^{\left(\sigma_{1}-\sigma\right)-\frac{1}{n}-1} d y \int_{0}^{1} \frac{(-\ln u)^{\beta}}{u^{\lambda}+1} u^{\sigma+\frac{1}{p n}-1} d u \\
= & I_{1} \leq M_{1} J_{1}=M_{1} n<\infty .
\end{aligned}
$$

Since $\left(\sigma_{1}-\sigma\right)-\frac{1}{n} \geq 0$, it follows that:

$$
\int_{1}^{\infty} y^{\left(\sigma_{1}-\sigma\right)-\frac{1}{n}-1} d y=\infty
$$

By (10), in view of:

$$
\int_{0}^{1} \frac{(-\ln u)^{\beta}}{u^{\lambda}+1} u^{\sigma+\frac{1}{p n}-1} d u>0,
$$

we deduce that $\infty<\infty$, which is a contradiction.

If $\sigma_{1}<\sigma$, then for $n \geq \frac{1}{\sigma-\sigma_{1}}(n \in \mathbf{N})$, we set the following two functions:

$$
\widetilde{f}_{n}(x):=\left\{\begin{array}{c}
0,0<x<1 \\
x^{\sigma-\frac{1}{p n}-1}, x \geq 1
\end{array}, \widetilde{g}_{n}(y):=\left\{\begin{array}{c}
y^{\sigma_{1}+\frac{1}{q^{n}}-1}, 0<y \leq 1 \\
0, y>1
\end{array},\right.\right.
$$

and obtain:

$$
\widetilde{J}_{1}:=\left[\int_{0}^{\infty} x^{p(1-\sigma)-1} \widetilde{f}_{n}^{p}(x) d x\right]^{\frac{1}{p}}\left[\int_{0}^{\infty} y^{q\left(1-\sigma_{1}\right)-1} \widetilde{g}_{n}^{q}(y) d y\right]^{\frac{1}{q}}=n .
$$

Setting $u=x y$, we obtain:

$$
\begin{aligned}
\widetilde{I}_{1} & :=\int_{0}^{\infty} \widetilde{f}_{n}(x)\left[\int_{0}^{\frac{1}{x}} \frac{|\ln x y|^{\beta}}{(x y)^{\lambda}+1} \widetilde{g}_{n}(y) d y\right] d x \\
& =\int_{1}^{\infty}\left[\int_{0}^{\frac{1}{x}} \frac{(-\ln x y)^{\beta}}{(x y)^{\lambda}+1} y^{\sigma_{1}+\frac{1}{q^{n}}-1} d y\right] x^{\sigma-\frac{1}{p^{n}}-1} d x \\
& =\int_{1}^{\infty} x^{\left(\sigma-\sigma_{1}\right)-\frac{1}{n}-1} d x \int_{0}^{1} \frac{(-\ln u)^{\beta}}{u^{\lambda}+1} u^{\sigma_{1}+\frac{1}{q^{n}}-1} d u
\end{aligned}
$$

and then by Fubini's theorem and (9), we have:

$$
\begin{aligned}
& \int_{1}^{\infty} x^{\left(\sigma-\sigma_{1}\right)-\frac{1}{n}-1} d x \int_{0}^{1} \frac{(-\ln u)^{\beta}}{u^{\lambda}+1} u^{\sigma_{1}+\frac{1}{q^{n}}-1} d u \\
= & \widetilde{I}_{1}=\int_{0}^{\infty} \widetilde{g}_{n}(y)\left[\int_{0}^{\frac{1}{y}} \frac{|\ln x y|^{\beta} \widetilde{f}_{n}(x)}{(x y)^{\lambda}+1} d x\right] d y \leq M_{1} \widetilde{J}_{1}=M_{1} n .
\end{aligned}
$$

Since $\left(\sigma-\sigma_{1}\right)-\frac{1}{n} \geq 0$, it follows that:

$$
\int_{1}^{\infty} x^{\left(\sigma-\sigma_{1}\right)-\frac{1}{n}-1} d x=\infty
$$

By (11), in view of the fact that 


$$
\int_{0}^{1} \frac{(-\ln u)^{\beta}}{u^{\lambda}+1} u^{\sigma_{1}+\frac{1}{q n}-1} d u>0,
$$

we obtain that $\infty<\infty$, which is a contradiction.

Hence, we conclude that $\sigma_{1}=\sigma$.

For $\sigma_{1}=\sigma$, we reduce (11) as follows:

$$
M_{1} \geq \int_{0}^{1} \frac{(-\ln u)^{\beta}}{u^{\lambda}+1} u^{\sigma+\frac{1}{q^{n}}-1} d u .
$$

Since:

$$
\left\{\frac{(-\ln u)^{\beta}}{u^{\lambda}+1} u^{\sigma+\frac{1}{q^{n}}-1}\right\}_{n=1}^{\infty}
$$

is non-negative and increasing in $(0,1]$, by Levi's theorem, we derive that:

$$
\begin{aligned}
M_{1} & \geq \lim _{n \rightarrow \infty} \int_{0}^{1} \frac{(-\ln u)^{\beta}}{u^{\lambda}+1} u^{\sigma+\frac{1}{q^{n}}-1} d u \\
& =\int_{0}^{1} \lim _{n \rightarrow \infty} \frac{(-\ln u)^{\beta}}{u^{\lambda}+1} u^{\sigma+\frac{1}{q^{n}}-1} d u=k_{1}(\sigma) .
\end{aligned}
$$

This completes the proof of the lemma.

Lemma 2. If $\beta>-1,0<\sigma<\lambda$, there exists a constant $M_{2}$, such that for any non-negative measurable functions $f(x)$ and $g(y)$ in $(0, \infty)$, the following inequality:

$$
\begin{aligned}
& \int_{0}^{\infty} g(y)\left[\int_{\frac{1}{y}}^{\infty} \frac{|\ln x y|^{\beta}}{(x y)^{\lambda}+1} f(x) d x\right] d y \\
\leq & M_{2}\left[\int_{0}^{\infty} x^{p(1-\sigma)-1} f^{p}(x) d x\right]^{\frac{1}{p}}\left[\int_{0}^{\infty} y^{q\left(1-\sigma_{1}\right)-1} g^{q}(y) d y\right]^{\frac{1}{q}}
\end{aligned}
$$

holds true. Then, we have $\sigma_{1}=\sigma$, and $M_{2} \geq k_{2}(\sigma)$.

Proof. If $\sigma_{1}<\sigma$, then for $n \geq \frac{1}{\sigma-\sigma_{1}}(n \in \mathbf{N})$, we set two functions $\widetilde{f}_{n}(x)$ and $\widetilde{g}_{n}(y)$ as in Lemma 1, and derive that:

$$
\widetilde{J}_{1}=\left[\int_{0}^{\infty} x^{p(1-\sigma)-1} \widetilde{f}_{n}^{p}(x) d x\right]^{\frac{1}{p}}\left[\int_{0}^{\infty} y^{q\left(1-\sigma_{1}\right)-1} \widetilde{g}_{n}^{q}(y) d y\right]^{\frac{1}{q}}=n .
$$

Setting $u=x y$, we obtain:

$$
\begin{aligned}
\widetilde{I}_{2} & :=\int_{0}^{\infty} \widetilde{g}_{n}(y)\left[\int_{\frac{1}{y}}^{\infty} \frac{|\ln x y|^{\beta}}{(x y)^{\lambda}+1} \widetilde{f}_{n}(x) d x\right] d y \\
& =\int_{0}^{1}\left[\int_{\frac{1}{y}}^{\infty} \frac{(\ln x y)^{\beta}}{(x y)^{\lambda}+1} x^{\sigma-\frac{1}{p n}-1} d x\right] y^{\sigma_{1}+\frac{1}{q^{n}}-1} d y \\
& =\int_{0}^{1} y^{\left(\sigma_{1}-\sigma\right)+\frac{1}{n}-1} d y \int_{1}^{\infty} \frac{(\ln u)^{\beta}}{u^{\lambda}+1} u^{\sigma-\frac{1}{p^{n}}-1} d u,
\end{aligned}
$$

and then by (13), we deduce that:

$$
\begin{aligned}
& \int_{0}^{1} y^{\left(\sigma_{1}-\sigma\right)+\frac{1}{n}-1} d y \int_{1}^{\infty} \frac{(\ln u)^{\beta}}{u^{\lambda}+1} u^{\sigma-\frac{1}{p n}-1} d u \\
= & \widetilde{I}_{2} \leq M_{2} \widetilde{J}_{1}=M_{2} n<\infty .
\end{aligned}
$$


Since $\left(\sigma_{1}-\sigma\right)+\frac{1}{n} \leq 0$, it follows that:

$$
\int_{0}^{1} y^{\left(\sigma_{1}-\sigma\right)+\frac{1}{n}-1} d y=\infty
$$

By (14), in view of

$$
\int_{1}^{\infty} \frac{(\ln u)^{\beta}}{u^{\lambda}+1} u^{\sigma-\frac{1}{p n}-1} d u>0,
$$

we have $\infty<\infty$, which is a contradiction.

If $\sigma_{1}>\sigma$, then for $n \geq \frac{1}{\sigma_{1}-\sigma}(n \in \mathbf{N})$, we set two sequences of $f_{n}(x)$ and $g_{n}(y)$ as in Lemma 1, and obtain:

$$
J_{1}=\left[\int_{0}^{\infty} x^{p(1-\sigma)-1} f_{n}^{p}(x) d x\right]^{\frac{1}{p}}\left[\int_{0}^{\infty} y^{q\left(1-\sigma_{1}\right)-1} g_{n}^{q}(y) d y\right]^{\frac{1}{q}}=n .
$$

Setting $u=x y$, we obtain:

$$
\begin{aligned}
I_{2} & :=\int_{0}^{\infty} f_{n}(x)\left[\int_{\frac{1}{x}}^{\infty} \frac{|\ln x y|^{\beta}}{(x y)^{\lambda}+1} g_{n}(y) d y\right] d x \\
& =\int_{0}^{1}\left[\int_{\frac{1}{x}}^{\infty} \frac{(\ln x y)^{\beta}}{(x y)^{\lambda}+1} y^{\sigma_{1}-\frac{1}{q n}-1} d y\right] x^{\sigma+\frac{1}{p n}-1} d x \\
& =\int_{0}^{1} x^{\left(\sigma-\sigma_{1}\right)+\frac{1}{n}-1} d x \int_{1}^{\infty} \frac{(\ln u)^{\beta}}{u^{\lambda}+1} u^{\sigma_{1}-\frac{1}{q n}-1} d u,
\end{aligned}
$$

and then, by Fubini's theorem and (13), we have:

$$
\begin{aligned}
& \int_{0}^{1} x^{\left(\sigma-\sigma_{1}\right)+\frac{1}{n}-1} d x \int_{1}^{\infty} \frac{(\ln u)^{\beta}}{u^{\lambda}+1} u^{\sigma_{1}-\frac{1}{q n}-1} d u \\
= & I_{2}=\int_{0}^{\infty} g_{n}(y)\left[\int_{\frac{1}{y}}^{\infty} \frac{|\ln x y|^{\beta} f_{n}(x)}{(x y)^{\lambda}+1} d x\right] d y \leq M_{2} J_{1}=M_{2} n .
\end{aligned}
$$

Since $\left(\sigma-\sigma_{1}\right)+\frac{1}{n} \leq 0$, it follows that

$$
\int_{0}^{1} x^{\left(\sigma-\sigma_{1}\right)+\frac{1}{n}-1} d x=\infty .
$$

By (15), in view of the fact that:

$$
\int_{1}^{\infty} \frac{(\ln u)^{\beta}}{u^{\lambda}+1} u^{\sigma_{1}-\frac{1}{q^{n}}-1} d u>0
$$

we have $\infty<\infty$, which is a contradiction.

Hence, we conclude the fact that $\sigma_{1}=\sigma$.

For $\sigma_{1}=\sigma$, we reduce (15) as follows:

$$
M_{2} \geq \int_{1}^{\infty} \frac{(\ln u)^{\beta}}{u^{\lambda}+1} u^{\sigma-\frac{1}{q n}-1} d u
$$

Since:

$$
\left\{\frac{(\ln u)^{\beta}}{u^{\lambda}+1} u^{\sigma-\frac{1}{q n}-1}\right\}_{n=1}^{\infty}
$$


is non-negative and increasing in $[1, \infty)$, still by Levi's theorem, we have:

$$
\begin{aligned}
M_{2} & \geq \lim _{n \rightarrow \infty} \int_{1}^{\infty} \frac{(\ln u)^{\beta}}{u^{\lambda}+1} u^{\sigma-\frac{1}{q^{n}}-1} d u \\
& =\int_{1}^{\infty} \lim _{n \rightarrow \infty} \frac{(\ln u)^{\beta}}{u^{\lambda}+1} u^{\sigma-\frac{1}{q^{n}}-1} d u=k_{2}(\sigma) .
\end{aligned}
$$

This completes the proof of the Lemma.

\section{Main Results and Corollaries}

Theorem 1. If $\beta>-1, \sigma, \lambda>0$, then the following conditions are equivalent.

(i) There exists a constant $M_{1}$, such that for any $f(x) \geq 0$, satisfying:

$$
0<\int_{0}^{\infty} x^{p(1-\sigma)-1} f^{p}(x) d x<\infty
$$

we have the following Hardy-type integral inequality of the first kind with nonhomogeneous kernel:

$$
\begin{aligned}
J & :=\left\{\int_{0}^{\infty} y^{p \sigma_{1}-1}\left[\int_{0}^{\frac{1}{y}} \frac{|\ln x y|^{\beta}}{(x y)^{\lambda}+1} f(x) d x\right]^{p} d y\right\}^{\frac{1}{p}} \\
& <M_{1}\left[\int_{0}^{\infty} x^{p(1-\sigma)-1} f^{p}(x) d x\right]^{\frac{1}{p}} .
\end{aligned}
$$

(ii) There exists a constant $M_{1}$, such that for any $f(x), g(y) \geq 0$, satisfying:

$$
0<\int_{0}^{\infty} x^{p(1-\sigma)-1} f^{p}(x) d x<\infty \text { and } 0<\int_{0}^{\infty} y^{q\left(1-\sigma_{1}\right)-1} g^{q}(y) d y<\infty,
$$

we have the following inequality:

$$
\begin{aligned}
I & :=\int_{0}^{\infty} g(y)\left[\int_{0}^{\frac{1}{y}} \frac{|\ln x y|^{\beta}}{(x y)^{\lambda}+1} f(x) d x\right] d y \\
& <M_{1}\left[\int_{0}^{\infty} x^{p(1-\sigma)-1} f^{p}(x) d x\right]^{\frac{1}{p}}\left[\int_{0}^{\infty} y^{q\left(1-\sigma_{1}\right)-1} g^{q}(y) d y\right]^{\frac{1}{q}} .
\end{aligned}
$$

(iii) $\sigma_{1}=\sigma$.

If Condition (iii) holds, then $M_{1} \geq k_{1}(\sigma)$ and the constant factor:

$$
M_{1}=k_{1}(\sigma)=\frac{\Gamma(\beta+1)}{\lambda^{\beta+1}} \xi\left(\beta+1, \frac{\sigma}{\lambda}\right)
$$

in (17) and (18) is the best possible.

Proof. $(i) \Rightarrow($ ii $)$. By Hölder's inequality (cf. [29,30]), we obtain:

$$
\begin{aligned}
I & =\int_{0}^{\infty}\left[y^{\sigma_{1}-\frac{1}{p}} \int_{0}^{\frac{1}{y}} \frac{|\ln x y|^{\beta}}{(x y)^{\lambda}+1} f(x) d x\right]\left(y^{\frac{1}{p}-\sigma_{1}} g(y)\right) d y \\
& \leq J\left[\int_{0}^{\infty} y^{q\left(1-\sigma_{1}\right)-1} g^{q}(y) d y\right]^{\frac{1}{q}} .
\end{aligned}
$$

Then by (17), we have (18).

$($ ii $) \Rightarrow($ iii $)$. By Lemma 1 , we have $\sigma_{1}=\sigma$. 
(iii) $\Rightarrow($ i). Setting $u=x y$, we obtain the following weight function:

$$
\begin{aligned}
\omega_{1}(\sigma, y) & :=y^{\sigma} \int_{0}^{\frac{1}{y}} \frac{|\ln x y|^{\beta}}{(x y)^{\lambda}+1} x^{\sigma-1} d x \\
& =\int_{0}^{1} \frac{(-\ln u)^{\beta}}{u^{\lambda}+1} u^{\sigma-1} d u=k_{1}(\sigma)(y>0) .
\end{aligned}
$$

By Hölder's inequality with weight and (20), for $y \in(0, \infty)$, we have:

$$
\begin{aligned}
& {\left[\int_{0}^{\frac{1}{y}} \frac{|\ln x y|^{\beta}}{(x y)^{\lambda}+1} f(x) d x\right]^{p} } \\
= & \left\{\int_{0}^{\frac{1}{y}} \frac{|\ln x y|^{\beta}}{(x y)^{\lambda}+1}\left[\frac{y^{(\sigma-1) / p}}{x^{(\sigma-1) / q}} f(x)\right]\left[\frac{x^{(\sigma-1) / q}}{y^{(\sigma-1) / p}}\right] d x\right\}^{p} \\
\leq & \int_{0}^{\frac{1}{y}} \frac{|\ln x y|^{\beta}}{(x y)^{\lambda}+1} \frac{y^{\sigma-1} f^{p}(x)}{x^{(\sigma-1) p / q}} d x\left[\int_{0}^{\frac{1}{y}} \frac{|\ln x y|^{\beta}}{\left|(x y)^{\lambda}-1\right|} \frac{x^{\sigma-1} d x}{y^{(\sigma-1) q / p}}\right]^{p-1} \\
= & {\left[\omega_{1}(\sigma, y) y^{q(1-\sigma)-1}\right]^{p-1} \int_{0}^{\frac{1}{y}} \frac{|\ln x y|^{\beta}}{(x y)^{\lambda}+1} \frac{y^{\sigma-1}}{x^{(\sigma-1) p / q}} f^{p}(x) d x } \\
= & \left(k_{1}(\sigma)\right)^{p-1} y^{-p \sigma+1} \int_{0}^{\frac{1}{y}} \frac{|\ln x y|^{\beta}}{\left|(x y)^{\lambda}-1\right|} \frac{y^{\sigma-1}}{x^{(\sigma-1) p / q}} f^{p}(x) d x .
\end{aligned}
$$

If (21) takes the form of equality for some $y \in(0, \infty)$, then (cf. [30]) there exist constants $A$ and $B$, such that they are not all zero and:

$$
A \frac{y^{\sigma-1}}{x^{(\sigma-1) p / q}} f^{p}(x)=B \frac{x^{\sigma-1}}{y^{(\sigma-1) q / p}} \text { a.e. in } \mathbf{R}_{+} .
$$

We suppose that $A \neq 0$ (otherwise $B=A=0$ ). It follows that:

$$
x^{p(1-\sigma)-1} f^{p}(x)=y^{q(1-\sigma)} \frac{B}{A x} \text { a.e. in } \mathbf{R}_{+},
$$

which contradicts the fact that:

$$
0<\int_{0}^{\infty} x^{p(1-\sigma)-1} f^{p}(x) d x<\infty .
$$

Hence, (21) takes the form of strict inequality.

For $\sigma_{1}=\sigma$, by (21) and Fubini's theorem, we obtain:

$$
\begin{aligned}
J & <\left(k_{1}(\sigma)\right)^{\frac{1}{q}}\left\{\int_{0}^{\infty}\left[\int_{0}^{\frac{1}{y}} \frac{|\ln x y|^{\beta}}{(x y)^{\lambda}+1} \frac{y^{\sigma-1}}{x^{(\sigma-1) p / q}} f^{p}(x) d x\right] d y\right\}^{\frac{1}{p}} \\
& =\left(k_{1}(\sigma)\right)^{\frac{1}{q}}\left\{\int_{0}^{\infty}\left[\int_{0}^{\frac{1}{x}} \frac{|\ln x y|^{\beta}}{(x y)^{\lambda}+1} \frac{y^{\sigma-1}}{x^{(\sigma-1)(p-1)}} d y\right] f^{p}(x) d x\right\}^{\frac{1}{p}} \\
& =\left(k_{1}(\sigma)\right)^{\frac{1}{q}}\left[\int_{0}^{\infty} \omega_{1}(\sigma, x) x^{p(1-\sigma)-1} f^{p}(x) d x\right]^{\frac{1}{p}} \\
& =k_{1}(\sigma)\left[\int_{0}^{\infty} x^{p(1-\sigma)-1} f^{p}(x) d x\right]^{\frac{1}{p}} .
\end{aligned}
$$

Setting $M_{1} \geq k_{1}(\sigma)$, (17) follows.

Therefore, Condition (i), Condition (ii) and Condition (iii) are equivalent. 
When Condition (iii) is satisfied, if there exists a constant factor $M_{1} \leq k_{1}(\sigma)$, such that (18) is valid, then by Lemma 1 we have $M_{1} \geq k_{1}(\sigma)$. Then, the constant factor $M_{1}=k_{1}(\sigma)$ in (18) is the best possible. The constant factor $M_{1}=k_{1}(\sigma)$ in (17) is still the best possible. Otherwise, by (19) (for $\sigma_{1}=\sigma$ ), we can conclude that the constant factor $M_{1}=k_{1}(\sigma)$ in (18) is not the best possible.

Setting $y=\frac{1}{Y}, G(Y)=Y^{\lambda-2} g\left(\frac{1}{Y}\right), \mu_{1}=\lambda-\sigma_{1}$ in Theorem 1 , then replacing $Y$ (resp. $G(Y))$ by $y$ (resp. $g(y)$ ), we derive the following Corollary.

Corollary 1. If $\beta>-1, \sigma, \lambda>0$, then the following conditions are equivalent.

(i) There exists a constant $M_{1}$, such that for any $f(x) \geq 0$, satisfying:

$$
0<\int_{0}^{\infty} x^{p(1-\sigma)-1} f^{p}(x) d x<\infty
$$

we have the following Hardy-type inequality of the first kind with homogeneous kernel:

$$
\begin{aligned}
& \left\{\int_{0}^{\infty} y^{p \mu_{1}-1}\left[\int_{0}^{y} \frac{|\ln (x / y)|^{\beta}}{x^{\lambda}+y^{\lambda}} f(x) d x\right]^{p} d y\right\}^{\frac{1}{p}} \\
< & M_{1}\left[\int_{0}^{\infty} x^{p(1-\sigma)-1} f^{p}(x) d x\right]^{\frac{1}{p}} .
\end{aligned}
$$

(ii) There exists a constant $M_{1}$, such that for any $f(x), g(y) \geq 0$, satisfying:

$$
0<\int_{0}^{\infty} x^{p(1-\sigma)-1} f^{p}(x) d x<\infty \text { and } 0<\int_{0}^{\infty} y^{q\left(1-\mu_{1}\right)-1} g^{q}(y) d y<\infty,
$$

we have the following inequality:

$$
\begin{aligned}
& \int_{0}^{\infty} g(y)\left[\int_{0}^{y} \frac{|\ln (x / y)|^{\beta}}{x^{\lambda}+y^{\lambda}} f(x) d x\right] d y \\
< & M_{1}\left[\int_{0}^{\infty} x^{p(1-\sigma)-1} f^{p}(x) d x\right]^{\frac{1}{p}}\left[\int_{0}^{\infty} y^{q\left(1-\mu_{1}\right)-1} g^{q}(y) d y\right]^{\frac{1}{q}} ;
\end{aligned}
$$

(iii) $\mu_{1}=\mu$.

If Condition (iii) holds, then we have $M_{1} \geq k_{1}(\sigma)$, and the constant $M_{1}=k_{1}(\sigma)$ in (22) and (23) is the best possible.

Similarly, we obtain the following weight function:

$$
\begin{aligned}
\omega_{2}(\sigma, y) & :=y^{\sigma} \int_{\frac{1}{y}}^{\infty} \frac{|\ln x y|^{\beta} x^{\sigma-1}}{(x y)^{\lambda}+1} d x \\
& =\int_{1}^{\infty} \frac{\ln ^{\beta} u}{u^{\lambda}+1} u^{\sigma-1} d u=k_{2}(\sigma)(y>0)
\end{aligned}
$$

and then in view of Lemma 2 and in a similar manner, we obtain the following theorem:

Theorem 2. If $\beta>-1,0<\sigma=\lambda-\mu<\lambda$, then the following conditions are equivalent.

(i) There exists a constant $M_{2}$, such that for any $f(x) \geq 0$, satisfying:

$$
0<\int_{0}^{\infty} x^{p(1-\sigma)-1} f^{p}(x) d x<\infty
$$


we have the following Hardy-type inequality of the second kind with the nonhomogeneous kernel:

$$
\begin{aligned}
& \left\{\int_{0}^{\infty} y^{p \sigma_{1}-1}\left[\int_{\frac{1}{y}}^{\infty} \frac{|\ln x y|^{\beta}}{(x y)^{\lambda}+1} f(x) d x\right]^{p} d y\right\}^{\frac{1}{p}} \\
< & M_{2}\left[\int_{0}^{\infty} x^{p(1-\sigma)-1} f^{p}(x) d x\right]^{\frac{1}{p}} .
\end{aligned}
$$

(ii) There exists a constant $M_{2}$, such that for any $f(x), g(y) \geq 0$, satisfying:

$$
0<\int_{0}^{\infty} x^{p(1-\sigma)-1} f^{p}(x) d x<\infty \text { and } 0<\int_{0}^{\infty} y^{q\left(1-\sigma_{1}\right)-1} g^{q}(y) d y<\infty,
$$

we have the following inequality:

$$
\begin{aligned}
& \int_{0}^{\infty} g(y)\left[\int_{\frac{1}{y}}^{\infty} \frac{|\ln x y|^{\beta}}{(x y)^{\lambda}+1} f(x) d x\right] d y \\
< & M_{2}\left[\int_{0}^{\infty} x^{p(1-\sigma)-1} f^{p}(x) d x\right]^{\frac{1}{p}}\left[\int_{0}^{\infty} y^{q\left(1-\sigma_{1}\right)-1} g^{q}(y) d y\right]^{\frac{1}{q}} .
\end{aligned}
$$

(iii) $\sigma_{1}=\sigma$.

If Condition (iii) holds, then we have $M_{2} \geq k_{2}(\sigma)$, and the constant factor:

$$
M_{2}=k_{2}(\sigma)=\frac{\Gamma(\beta+1)}{\lambda^{\beta+1}} \xi\left(\beta+1, \frac{\mu}{\lambda}\right)=k_{1}(\mu)
$$

in (24) and (25) is the best possible.

Setting:

$$
y=\frac{1}{Y}, G(Y)=Y^{\lambda-2} g\left(\frac{1}{Y}\right), \mu_{1}=\lambda-\sigma_{1}
$$

in Theorem 2, then replacing $Y$ (resp. $G(Y)$ ) by $y$ (resp. $g(y)$ ), we derive the following Corollary.

Corollary 2. If $\beta>-1,0<\sigma=\lambda-\mu<\lambda$, then the following conditions are equivalent.

(i) There exists a constant $M_{2}$, such that for any $f(x) \geq 0$, satisfying:

$$
0<\int_{0}^{\infty} x^{p(1-\sigma)-1} f^{p}(x) d x<\infty
$$

we have the following Hardy-type inequality of the second kind with homogeneous kernel:

$$
\begin{aligned}
& \left\{\int_{0}^{\infty} y^{p \mu_{1}-1}\left[\int_{y}^{\infty} \frac{|\ln (x / y)|^{\beta}}{x^{\lambda}+y^{\lambda}} f(x) d x\right]^{p} d y\right\}^{\frac{1}{p}} \\
< & M_{2}\left[\int_{0}^{\infty} x^{p(1-\sigma)-1} f^{p}(x) d x\right]^{\frac{1}{p}} ;
\end{aligned}
$$

(ii) There exists a constant $M_{2}$, such that for any $f(x), g(y) \geq 0$, satisfying

$$
0<\int_{0}^{\infty} x^{p(1-\sigma)-1} f^{p}(x) d x<\infty \text { and } 0<\int_{0}^{\infty} y^{q\left(1-\mu_{1}\right)-1} g^{q}(y) d y<\infty,
$$


we have the following inequality:

$$
\begin{aligned}
& \int_{0}^{\infty} g(y)\left[\int_{y}^{\infty} \frac{|\ln (x / y)|^{\beta}}{x^{\lambda}+y^{\lambda}} f(x) d x\right] d y \\
< & M_{2}\left[\int_{0}^{\infty} x^{p(1-\sigma)-1} f^{p}(x) d x\right]^{\frac{1}{p}}\left[\int_{0}^{\infty} y^{q\left(1-\mu_{1}\right)-1} g^{q}(y) d y\right]^{\frac{1}{q}} .
\end{aligned}
$$

(iii) $\mu_{1}=\mu$.

If Condition (iii) holds, then we have $M_{2} \geq k_{2}(\sigma)$, and the constant $M_{2}=k_{2}(\sigma)=k_{1}(\mu)$ in (26) and (27) is the best possible.

\section{Operator Expressions}

For $\sigma, \lambda>0, \mu=\lambda-\sigma$, we set the following functions:

$$
\varphi(x):=x^{p(1-\sigma)-1}, \quad \psi(y):=y^{q(1-\sigma)-1}, \phi(y):=y^{q(1-\mu)-1},
$$

and:

$$
\psi^{1-p}(y)=y^{p \sigma-1}, \phi^{1-p}(y)=y^{p \mu-1}\left(x, y \in \mathbf{R}_{+}\right) .
$$

Define the following real normed linear spaces:

$$
\begin{aligned}
L_{p, \varphi}\left(\mathbf{R}_{+}\right) & :=\left\{f:|| f \|_{p, \varphi}:=\left(\int_{0}^{\infty} \varphi(x)|f(x)|^{p} d x\right)^{\frac{1}{p}}<\infty\right\}, \\
L_{q, \psi}\left(\mathbf{R}_{+}\right) & =\left\{g:\|g\|_{q, \psi}:=\left(\int_{0}^{\infty} \psi(y)|g(y)|^{q} d y\right)^{\frac{1}{q}}<\infty\right\}, \\
L_{q, \phi}\left(\mathbf{R}_{+}\right) & =\left\{g:\|g\|_{q, \phi}:=\left(\int_{0}^{\infty} \phi(y)|g(y)|^{q} d y\right)^{\frac{1}{q}}<\infty\right\}, \\
L_{p, \psi^{1-p}}\left(\mathbf{R}_{+}\right) & =\left\{h:\|h\|_{p, \psi^{1-p}}=\left(\int_{0}^{\infty} \psi^{1-p}(y)|h(y)|^{p} d y\right)^{\frac{1}{p}}<\infty\right\}, \\
L_{q, \phi^{1-p}}\left(\mathbf{R}_{+}\right) & =\left\{h:\|h\|_{p, \phi^{1-p}}=\left(\int_{0}^{\infty} \phi^{1-p}(y)|h(y)|^{p} d y\right)^{\frac{1}{p}}<\infty\right\} .
\end{aligned}
$$

(a) In view of Theorem 1 (setting $\sigma_{1}=\sigma$ ), for $f \in L_{p, \varphi}\left(\mathbf{R}_{+}\right)$, setting:

$$
h_{1}(y):=\int_{0}^{\frac{1}{y}} \frac{|\ln x y|^{\beta}}{(x y)^{\lambda}+1} f(x) d x\left(y \in \mathbf{R}_{+}\right) \text {, }
$$

by (17), we have:

$$
\left\|h_{1}\right\|_{p, \psi^{1-p}}=\left[\int_{0}^{\infty} \psi^{1-p}(y) h_{1}^{p}(y) d y\right]^{\frac{1}{p}}<M_{1}\|f\|_{p, \varphi}<\infty .
$$

Definition 1. Define a Hardy-type integral operator of the first kind with the nonhomogeneous kernel:

$$
T_{1}^{(1)}: L_{p, \varphi}\left(\mathbf{R}_{+}\right) \rightarrow L_{p, \psi^{1-p}}\left(\mathbf{R}_{+}\right)
$$

as follows.

For any $f \in L_{p, \varphi}\left(\mathbf{R}_{+}\right)$, there exists a unique representation:

$$
T_{1}^{(1)} f=h_{1} \in L_{p, \psi^{1-p}}\left(\mathbf{R}_{+}\right),
$$


satisfying $T_{1}^{(1)} f(y)=h_{1}(y)$, for any $y \in \mathbf{R}_{+}$.

In view of (28), it follows that:

$$
\left\|T_{1}^{(1)} f\right\|_{p, \psi^{1-p}}=\left\|h_{1}\right\|_{p, \psi^{1-p}} \leq M_{1}\|f\|_{p, \varphi},
$$

and then the operator $T_{1}^{(1)}$ is bounded satisfying

$$
\left\|T_{1}^{(1)}\right\|=\sup _{f(\neq 0) \in L_{p, \varphi}\left(\mathbf{R}_{+}\right)} \frac{\left\|T_{1}^{(1)} f\right\|_{p, \psi^{1-p}}}{\|f\|_{p, \varphi}} \leq M_{1} .
$$

If we define the formal inner product of $T_{1}^{(1)} f$ and $g$ as follows:

$$
\left(T_{1}^{(1)} f, g\right):=\int_{0}^{\infty}\left[\int_{0}^{\frac{1}{y}} \frac{|\ln x y|^{\beta}}{(x y)^{\lambda}+1} f(x) d x\right] g(y) d y,
$$

then we can rewrite Theorem 1 as follows.

Theorem 3. For $\beta>-1, \sigma, \lambda>0$, the following conditions are equivalent.

(i) There exists a constant $M_{1}$, such that for any $f(x) \geq 0, f \in L_{p, \varphi}\left(\mathbf{R}_{+}\right),\|f\|_{p, \varphi}>0$, we have the following inequality:

$$
\left\|T_{1}^{(1)} f\right\|_{p, \psi^{1-p}}<M_{1}\|f\|_{p, \varphi} .
$$

(ii) There exists a constant $M_{1}$, such that for any $f(x), g(y) \geq 0, f \in L_{p, \varphi}\left(\mathbf{R}_{+}\right), g \in L_{q, \psi}\left(\mathbf{R}_{+}\right)$, $\|f\|_{p, \varphi},\|g\|_{q, \psi}>0$, we have the following inequality:

$$
\left(T_{1}^{(1)} f, g\right)<M_{1}\|f\|_{p, \varphi}\|g\|_{q, \psi} .
$$

We also have that $\left\|T_{1}^{(1)}\right\|=k_{1}(\sigma) \leq M_{1}$.

(b) In view of Corollary 1 (setting $\mu_{1}=\mu$ ), for $f \in L_{p, \varphi}\left(\mathbf{R}_{+}\right)$, considering the function:

$$
h_{2}(y):=\int_{0}^{y} \frac{|\ln (x / y)|^{\beta}}{x^{\lambda}+y^{\lambda}} f(x) d x\left(y \in \mathbf{R}_{+}\right) \text {, }
$$

by (22), we have:

$$
\left\|h_{2}\right\|_{p, \phi^{1-p}}=\left[\int_{0}^{\infty} \phi^{1-p}(y) h_{2}^{p}(y) d y\right]^{\frac{1}{p}}<M_{1}\|f\|_{p, \varphi}<\infty .
$$

Definition 2. Define a Hardy-type integral operator of the first kind with the homogeneous kernel:

$$
T_{1}^{(2)}: L_{p, \varphi}\left(\mathbf{R}_{+}\right) \rightarrow L_{p, \phi^{1-p}}\left(\mathbf{R}_{+}\right)
$$

as follows.

For any $f \in L_{p, \varphi}(\mathbf{R})$, there exists a unique representation:

$$
T_{1}^{(2)} f=h_{2} \in L_{p, \phi^{1-p}}\left(\mathbf{R}_{+}\right)
$$

satisfying $T_{1}^{(2)} f(y)=h_{2}(y)$, for any $y \in \mathbf{R}_{+}$.

In view of (31), it follows that: 


$$
\left\|T_{1}^{(2)} f\right\|_{p, \phi^{1-p}}=\left\|h_{2}\right\|_{p, \phi^{1-p}} \leq M_{1}\|f\|_{p, \varphi}
$$

and then the operator $T_{1}^{(2)}$ is bounded satisfying:

$$
\left\|T_{1}^{(2)}\right\|=\sup _{f(\neq 0) \in L_{p, \varphi}\left(\mathbf{R}_{+}\right)} \frac{\left\|T_{1}^{(2)} f\right\|_{p, \phi^{1-p}}}{\|f\|_{p, \varphi}} \leq M_{1} .
$$

If we define the formal inner product of $T_{1}^{(2)} f$ and $g$ as follows:

$$
\left(T_{1}^{(2)} f, g\right):=\int_{0}^{\infty}\left[\int_{0}^{y} \frac{|\ln (x / y)|^{\beta}}{x^{\lambda}+y^{\lambda}} f(x) d x\right] g(y) d y,
$$

then we can rewrite Corollary 1 as follows.

Corollary 3. For $\beta>-1, \sigma, \lambda>0$, the following conditions are equivalent.

(i) There exists a constant $M_{1}$, such that for any $f(x) \geq 0, f \in L_{p, \varphi}\left(\mathbf{R}_{+}\right),\|f\|_{p, \varphi}>0$, we have the following inequality:

$$
\left\|T_{1}^{(2)} f\right\|_{p, \phi^{1-p}}<M_{1}\|f\|_{p, \varphi} .
$$

(ii) There exists a constant $M_{1}$, such that for any $f(x), g(y) \geq 0, f \in L_{p, \varphi}\left(\mathbf{R}_{+}\right), g \in L_{q, \phi}\left(\mathbf{R}_{+}\right)$, $\|f\|_{p, \varphi},\|g\|_{q, \phi}>0$, we have the following inequality:

$$
\left(T_{1}^{(2)} f, g\right)<M_{1}\|f\|_{p, \varphi}\|g\|_{q, \phi} .
$$

We still have $\left\|T_{1}^{(2)}\right\|=k_{1}(\sigma) \leq M_{1}$.

(c) In view of Theorem 2 (setting $\sigma_{1}=\sigma$ ), for $f \in L_{p, \varphi}\left(\mathbf{R}_{+}\right)$, considering the function:

$$
H_{1}(y):=\int_{\frac{1}{y}}^{\infty} \frac{|\ln x y|^{\beta}}{(x y)^{\lambda}+1} f(x) d x\left(y \in \mathbf{R}_{+}\right),
$$

by (24), we have:

$$
\left\|H_{1}\right\|_{p, \psi^{1-p}}=\left[\int_{0}^{\infty} \psi^{1-p}(y) H_{1}^{p}(y) d y\right]^{\frac{1}{p}}<M_{2}\|f\|_{p, \varphi}<\infty .
$$

(A. Raigorodskii)

Definition 3. Define a Hardy-type integral operator of the second kind with the nonhomogeneous kernel:

$$
T_{2}^{(1)}: L_{p, \varphi}\left(\mathbf{R}_{+}\right) \rightarrow L_{p, \psi^{1-p}}\left(\mathbf{R}_{+}\right)
$$

as follows.

For any $f \in L_{p, \varphi}\left(\mathbf{R}_{+}\right)$, there exists a unique representation:

$$
T_{2}^{(1)} f=H_{1} \in L_{p, \psi^{1-p}}\left(\mathbf{R}_{+}\right),
$$

satisfying $T_{2}^{(1)} f(y)=H_{1}(y)$, for any $y \in \mathbf{R}_{+}$.

In view of (34), it follows that:

$$
\left\|T_{2}^{(1)} f\right\|_{p, \psi^{1-p}}=\left\|H_{1}\right\|_{p, \psi^{1-p}} \leq M_{2}|| f \|_{p, \varphi},
$$


and then the operator $T_{2}^{(1)}$ is bounded satisfying:

$$
\left\|T_{2}^{(1)}\right\|=\sup _{f(\neq 0) \in L_{p, \varphi}\left(\mathbf{R}_{+}\right)} \frac{\left\|T_{2}^{(1)} f\right\|_{p, \psi^{1-p}}}{\|f\|_{p, \varphi}} \leq M_{2} .
$$

If we define the formal inner product of $T_{2}^{(1)} f$ and $g$ as follows.

$$
\left(T_{2}^{(1)} f, g\right):=\int_{0}^{\infty}\left[\int_{\frac{1}{y}}^{\infty} \frac{(\ln x y)^{\beta}}{(x y)^{\lambda}+1} f(x) d x\right] g(y) d y,
$$

then we can rewrite Theorem 2 as follows.

Theorem 4. For $\beta>-1,0<\sigma=\lambda-\mu<\lambda$, the following conditions are equivalent.

(i) There exists a constant $M_{2}$, such that for any $f(x) \geq 0, f \in L_{p, \varphi}\left(\mathbf{R}_{+}\right),\|f\|_{p, \varphi}>0$, we have the following inequality:

$$
\left\|T_{2}^{(1)} f\right\|_{p, \psi^{1-p}}<M_{2}\|f\|_{p, \varphi}
$$

(ii) There exists a constant $M_{2}$, such that for any $f(x), g(y) \geq 0, f \in L_{p, \varphi}\left(\mathbf{R}_{+}\right), g \in L_{q, \psi}\left(\mathbf{R}_{+}\right)$, $\|f\|_{p, \varphi},\|g\|_{q, \psi}>0$, we have the following inequality:

$$
\left(T_{2}^{(1)} f, g\right)<M_{2}\|f\|_{p, \varphi}\|g\|_{q, \psi} .
$$

We still have $\left\|T_{2}^{(1)}\right\|=k_{2}(\sigma) \leq M_{2}$.

(d) In view of Corollary 2 (setting $\mu_{1}=\mu$ ), for $f \in L_{p, \varphi}\left(\mathbf{R}_{+}\right)$, considering the function:

$$
H_{2}(y):=\int_{y}^{\infty} \frac{|\ln (x / y)|^{\beta}}{x^{\lambda}+y^{\lambda}} f(x) d x\left(y \in \mathbf{R}_{+}\right),
$$

by (26), we have:

$$
\left\|H_{2}\right\|_{p, \phi^{1-p}}=\left[\int_{0}^{\infty} \phi^{1-p}(y) H_{2}^{p}(y) d y\right]^{\frac{1}{p}}<M_{2}\|f\|_{p, \varphi}<\infty .
$$

Definition 4. Define a Hardy-type integral operator of the second kind with the homogeneous kernel:

$$
T_{2}^{(2)}: L_{p, \varphi}\left(\mathbf{R}_{+}\right) \rightarrow L_{p, \phi^{1-p}}\left(\mathbf{R}_{+}\right)
$$

as follows.

For any $f \in L_{p, \varphi}(\mathbf{R})$, there exists a unique representation:

$$
T_{2}^{(2)} f=H_{2} \in L_{p, \phi^{1-p}}\left(\mathbf{R}_{+}\right)
$$

satisfying $T_{2}^{(2)} f(y)=H_{2}(y)$, for any $y \in \mathbf{R}_{+}$.

In view of (37), it follows that:

$$
\left\|T_{2}^{(2)} f\right\|_{p, \phi^{1-p}}=\left\|H_{2}\right\|_{p, \phi^{1-p}} \leq M_{2}\|f\|_{p, \varphi},
$$


and then the operator $T_{2}^{(2)}$ is bounded satisfying.

$$
\left\|T_{2}^{(2)}\right\|=\sup _{f(\neq 0) \in L_{p, \varphi}\left(\mathbf{R}_{+}\right)} \frac{\left\|T_{2}^{(2)} f\right\|_{p, \phi^{1-p}}}{\|f\|_{p, \varphi}} \leq M_{2} .
$$

If we define the formal inner product of $T_{1}^{(2)} f$ and $g$ as follows:

$$
\left(T_{2}^{(2)} f, g\right):=\int_{0}^{\infty}\left[\int_{y}^{\infty} \frac{[\ln (x / y)]^{\beta}}{x^{\lambda}+y^{\lambda}} f(x) d x\right] g(y) d y,
$$

then we can rewrite Corollary 2 as follows.

Corollary 4. For $\beta>-1,0<\sigma=\lambda-\mu<\lambda$, the following conditions are equivalent.

(i) There exists a constant $M_{2}$, such that for any $f(x) \geq 0, f \in L_{p, \varphi}\left(\mathbf{R}_{+}\right),\|f\|_{p, \varphi}>0$, we have the following inequality:

$$
\left\|T_{2}^{(2)} f\right\|_{p, \phi^{1-p}}<M_{2}\|f\|_{p, \varphi} .
$$

(ii) There exists a constant $M_{2}$, such that for any $f(x), g(y) \geq 0, f \in L_{p, \varphi}\left(\mathbf{R}_{+}\right), g \in$ $L_{q, \phi}\left(\mathbf{R}_{+}\right),\|f\|_{p, \varphi},\|g\|_{q, \phi}>0$, we have the following inequality:

$$
\left(T_{2}^{(2)} f, g\right)<M_{2}\|f\|_{p, \varphi}\|g\|_{q, \phi} .
$$

We still have $\left\|T_{2}^{(2)}\right\|=k_{2}(\sigma)=k_{1}(\mu) \leq M_{2}$.

Author Contributions: Writing—original draft, M.T.R., B.Y. and A.R. All three authors contributed equally in all stages of preparation of this work. All authors have read and agreed to the published version of the manuscript.

Funding: This work was supported by the National Natural Science Foundation (No. 61772140), the Characteristic Innovation Project of Guangdong Provincial Colleges and universities in 2020 (No. 2020KTSCX088) and the support of megagrant number 075-15-2019-1926 and the grant "Leading scientific schools" number NSh-2540.2020.1 (075-15-2020-417).

Institutional Review Board Statement: Not applicable.

Informed Consent Statement: Not applicable.

Data Availability Statement: Not applicable.

Acknowledgments: B. C. Yang: This work was supported by the National Natural Science Foundation (No. 61772140) and the Characteristic Innovation Project of Guangdong Provincial Colleges and universities in 2020 (No. 2020KTSCX088). We are grateful for this help. A. Raigorodskii: This author acknowledges the Russian Federation Government for the financial support of his study: his research on these results was carried out with the support of megagrant number 075-15-2019-1926. His research on these results was also supported in the framework of the grant "Leading scientific schools" number NSh-2540.2020.1 (075-15-2020-417).

Conflicts of Interest: The authors declare no conflict of interest.

\section{References}

1. Hardy, G.H. Note on a theorem of Hilbert concerning series of positive terms. Proc. Lond. Math. Soc. 1925, 23, 45-46.

2. Schur, I. Bernerkungen sur Theorie der beschrankten Billnearformen mit unendlich vielen Veranderlichen. J. Math. 1911, 140, 1-28.

3. Hardy, G.H.; Littlewood, J.E.; Pólya, G. Inequalities; Cambridge University Press: Cambridge, MA, USA, 1934.

4. Mitrinović, D.S.; Pečarić, J.E.; Fink, A.M. Inequalities Involving Functions and Their Integrals and Deivatives; Kluwer Academic: Boston, MA, USA, 1991.

5. Yang, B.C. On Hilbert's integral inequality. J. Math. Anal. Appl. 1998, 220, 778-785. 
6. Yang, B.C. A note on Hilbert's integral inequality. Chin. Q. J. Math. 1998, 13, 83-86.

7. Yang, B.C. On an extension of Hilbert's integral inequality with some parameters. Aust. J. Math. Anal. Appl. $2004,1,11$.

8. Yang, B.C.; Brnetić, I.; Krnić, M.; Pečarić, J.E. Generalization of Hilbert and Hardy-Hilbert integral inequalities. Math. Ineq. Appl. 2005, 8, 259-272.

9. Krnić, M.; Pečarić, J.E. Hilbert's inequalities and their reverses. Publ. Math. Debr. 2005, 67, 315-331.

10. Hong, Y. On Hardy-Hilbert integral inequalities with some parameters. J. Ineq. Pure Appl. Math. $2005,6,92$.

11. Benyi, A.; Oh, C. Best constant for certain multi linear integral operator. J. Inequal. Appl. 2006, 2006, 28582. [CrossRef]

12. Li, Y.J.; He, B. On inequalities of Hilbert's type. Bull. Aust. Math. Soc. 2007, 76, 1-13. [CrossRef]

13. Zhong, W.Y.; Yang, B.C. On multiple Hardy-Hilbert's integral inequality with kernel. J. Ineq. Appl. 2007, 2007, 27962. [CrossRef]

14. Xu, J.S. Hardy-Hilbert's Inequalities with two parameters. Adv. Math. 2007, 36, 63-76.

15. Yang, B.C. The Norm of Operator and Hilbert-Type Inequalities; Science Press: Beijing, China, 2009.

16. Yang, B.C. Hilbert-Type Integral Inequalities; Bentham Science Publishers Ltd.: Sharjah, United Arab Emirates, 2009.

17. Yang, B.C. On Hilbert-type integral inequalities and their operator expressions. J. Guangdong Univ. Edu. 2013, 33, 1-17.

18. Hong, Y. On the structure character of Hilbert's type integral inequality with homogeneous kernal and applications. J. Jilin Univ. 2017, 55, 189-194.

19. Rassias, M.T.; Yang, B.C. Equivalent properties of a Hilbert-type integral inequality with the best constant factor related the Hurwitz zeta function. Ann. Funct. Anal. 2018, 9, 282-295. [CrossRef]

20. Rassias, M.T.; Yang, B.C.; Raigorodskii, A. Two kinds of the reverse Hardy-type integral inequalities with the equivalent forms related to the extended Riemann zeta function. Appl. Anal. Discret. Math. 2018, 12, 273-296. [CrossRef]

21. Gao, P. On weight Hardy inequalities for non-increasing sequence. J. Math. Ineq. 2018, 12, 551-557. [CrossRef]

22. Rassias, M.T.; Yang, B.C. On an equivalent property of a reverse Hilbert-type integral inequality related to the extended Hurwitz-zeta function. J. Math. Ineq. 2019, 13, 315-334. [CrossRef]

23. Rassias, M.T.; Yang, B.C. A reverse Mulholland-type inequality in the whole plane with multi-parameters. Appl. Anal. Discret. Math. 2019, 13, 290-308. [CrossRef]

24. You, M.H.; Guan, Y. On a Hilbert-type integral inequality with non-homogeneous kernel of mixed hyperbolic functions. J. Math. Ineq. 2019, 13, 1197-1208. [CrossRef]

25. Liu, Q. A Hilbert-type integral inequality under configuring free power and its applications. J. Ineq. Appl. 2019, 2019, 91. [CrossRef]

26. Chen, Q.; He, B.; Hong, Y.; Zhen, L. Equivalent parameter conditions for the validity of half-discrete Hilbert-type multiple integral inequality with generalized homogeneous kernel. J. Func. Spac. 2020, 2020, 7414861. [CrossRef]

27. Rassias, M.T.; Yang, B.C.; Raigorodskii, A. On Hardy-type integral inequality in the whole plane related to the extended Hurwitz-zeta fanction. J. Ineq. Appl. 2020, 2020, 94. [CrossRef]

28. Rassias, M.T.; Yang, B.C.; Raigorodskii, A. On the reverse Hardy-type integral inequalities in the whole plane with the extended Riemann-Zeta function. J. Math. Ineq. 2020, 14, 525-546. [CrossRef]

29. Wang, Z.Q.; Guo, D.R. Introduction to Special Functions; Science Press: Beijing, China, 1979.

30. Kuang, J.C. Applied Inequalities; Shangdong Science and Technology Press: Jinan, China, 2004. 